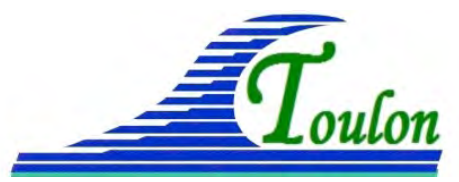

XIV èmes Journées Nationales Génie Côtier - Génie Civil

Toulon, 29 juin au $1^{\text {er }}$ juillet 2016

DOI:10.5150/jngcgc.2016.014 (C) Editions Paralia CFL

disponible en ligne - http://www.paralia.fr - available online

\title{
Modélisation du transport particulaire pour les énergies marines renouvelables
}

\author{
Feddy ADONG ${ }^{1}$, Anne-Claire BENNIS ${ }^{1}$, Dominique MOUAZE ${ }^{1}$ \\ 1. Université de Caen Normandie, UNICAEN, UMR M2C (UCBN, UR, CNRS-6143), \\ 24 rue des Tilleuls, 14000 Caen cedex 5, France.feddy.adong@unicaen.fr
}

\section{Résumé:}

La transition énergétique est au cœur de l'actualité avec notamment le développement des énergies marines renouvelables. Le dimensionnement et le positionnement des engins récupérateurs d'énergie nécessitent une bonne connaissance de l'environnement hydro-sédimentaire. Les sédiments en suspension peuvent affecter le fonctionnement des machines, en particulier dans un environnement hydrodynamique extrême comme celui du Raz-Blanchard. Afin de modéliser ce transport en suspension, un modèle d'éléments discrets simulant les sédiments a été forcé par un modèle hydrodynamique côtier. Des modifications ont été apportées au modèle d'éléments discrets pour prendre en compte le caractère tridimensionnel de l'écoulement ainsi que sa nature turbulente. L'étape de validation a été réalisée avec des expériences en laboratoire décrivant la mise en mouvement d'un lit sédimentaire par un écoulement fluide. Globalement, les résultats numériques sont en accord avec les mesures tant pour les profils verticaux de la vitesse fluide que pour la fraction solide. Ensuite, les premières simulations du transport sédimentaire de cailloutis pour un site côtier du Nord Cotentin sont discutées.

Mots-clés : Energies marines renouvelables, Modélisation hydro-sédimentaire, Transport particulaire.

\section{Introduction}

Disposant de plus de onze millions de $\mathrm{km}^{2}$ de zone marine sous sa juridiction, la France possède un fort potentiel d'exploitation des Énergies Marines Renouvelables (EMR). La Normandie, de par son potentiel éolien et hydrolien, est fortement concernée par les problèmes liés aux EMR. En 2020, les parcs éoliens de Courseulles sur mer, de Fécamp et de Dièppe-Tréport, ainsi que les fermes pilotes hydroliennes du Raz Blanchard seront mis en service. Ces installations seront exploitées pendant 20 à 30 ans à des fins commerciales.

Afin de préserver les machines récupératrices d'énergie et de minimiser les opérations de maintenance, il est important de comprendre l'impact des sédiments pour des problèmes liés à l'abrasion ou à la protection des systèmes contre les chocs. Des cailloutis qui se déplaceraient à grande vitesse dans un environnement mégatidal extrême comme celui du Raz-Blanchard pourraient causer beaucoup de dégâts en cas d'impacts avec les machines. Les conditions d'expérimentation étant très difficiles dans 


\section{Thème 2 - Dynamique sédimentaire}

les zones hydroliennes, l'outil numérique est incontournable pour étudier ces problèmes.

Deux approches permettent de modéliser puis de simuler le déplacement des sédiments marins. La première utilise une équation de transport contenant différentes paramétrisations représentant les processus opérants. De par la nature de l'équation résolue, cette approche modélise le déplacement des flux sédimentaires, et en particulier les phénomènes de dépôt et d'érosion, mais ne permet pas la retranscription des différents contacts entre les sédiments ou avec une structure immergée. La seconde méthode permet de résoudre le transport sédimentaire à l'échelle du sédiment, ce qui permet de prendre en compte les interactions entre les sédiments mais aussi les interactions entre les sédiments et les structures.

Nous proposons ici d'utiliser la deuxième méthode et de forcer le mouvement des sédiments par les courants calculés par un modèle hydrodynamique côtier pour une bathymétrie réaliste. Ainsi, nous mettons en œuvre le forçage du m odèle d'éléments discrets YADE (ŠMILAUER et al., 2010) simulant chaque sédiment par le modèle hydrodynamique côtier MARS3D (LAZURE \& DUMAS, 2008).

La section 2 est consacrée à la modélisation granulaire, avec la description de YADE et des modifications effectuées pour le forçage par MARS3D (MARS pour la suite), et de leurs validations. La section 3 illustre l'application au site du Nord Cotentin avec des premières simulations en environnement mégatidal extrême. Enfin, nous conclurons dans la section 4 .

\section{Modélisation granulaire}

\subsection{Méthode}

YADE est utilisé pour l'étude des déplacements et des interactions des sédiments. Ce modèle est basé sur la méthode des éléments discrets (DEM) qui assure un s uivi Lagrangien de chaque sédiment avec la prise en compte des forces de contact. Dans cette méthode, la position du sédiment est obtenue par résolution de la seconde loi de Newton où les forces appliquées au sédiment sont: les forces de contact, la force de gravité et les forces exercées par le fluide.

Le module HydroForceEngine présent dans le noyau de YADE assure le forçage de YADE par un écoulement unidirectionnel (MAURIN et al., 2015). Dans ce module, les forces exercées par le fluide sur le sédiment $\left(\overrightarrow{F_{f}}\right)$ se décomposent comme :

$\overrightarrow{F_{f}}=\overrightarrow{F_{d}}+\overrightarrow{F_{l}}+\overrightarrow{F_{b}}$

où $\overrightarrow{F_{d}}$ est la force de traînée, $\overrightarrow{F_{l}}$ est la force de portance et $\overrightarrow{F_{b}}$ la force de flottabilité. Les expressions de $\overrightarrow{F_{b}}$ et $\overrightarrow{F_{l}}$ données dans WIBERG \& SMITH (1985) sont conservées. L'expression de $\overrightarrow{F_{d}}$ est remplacée par une expression empirique proposée par DI FELICE (1994) car plus adaptée à des écoulements à fort nombre de Reynolds de 


\section{XII ${ }^{\text {èmes }}$ Journées Nationales Génie Côtier - Génie Civil \\ Cherbourg, 12-14 juin 2012}

particule. Afin de forcer YADE par MARS, nous avons mis en place une généralisation 3D du module HydroForceEngine et introduit la coordonnée verticale sigma, déjà présente dans MARS, définie comme :

$\sigma_{w}=\frac{z-x_{e}}{h_{0}+\mathrm{x}_{e}}, \quad-1 \leq \sigma_{w} \leq 0$

où $-h_{0} \leq z \leq x_{\theta}$ est la coordonnée verticale naturelle, $x_{\theta}$ est l'élévation de la surface libre et $h_{0}$ est la profondeur. La possibilité d'utiliser une discrétisation verticale non uniforme a également été implémentée (i.e. raffinement près du fond).

\subsection{Validation}

Nous présentons ici la validation des modifications apportées à YADE à l'aide des résultats obtenus en laboratoire par AUSSILLOUS et al. (2003). L'objectif est de s'assurer de la validité des modifications apportées au module HydroForceEngine avant le forçage de YADE par MARS, qui est l'objet de la section 3. Nous commençons par donner une description rapide de l'expérience que nous désirons reproduire numériquement (pour plus détails voir AUSSILLOUS et al. (2013)).

Un lit sédimentaire formé de sphères de diamètre dp, de densité $\rho_{p}$ est soumis à un écoulement laminaire fortement visqueux. Les sphères sont partiellement disposées dans un tube de verre de hauteur $6.5 \mathrm{~cm}$, de largeur $3.5 \mathrm{~cm}$ et de longueur $100 \mathrm{~cm}$. L'épaisseur de la couche fluide ne contenant aucune sphère $h_{f}$ ainsi que celle de la couche granulaire en mouvement $h_{m}$ sont enregistrées toutes les $5 \mathrm{~s}$. Les paramètres physiques sont inventoriés dans le tableau 1 et correspondent au run2 de l'étude expérimentale. Les sphères sont composées de borosilicate.

Tableau 1. Paramètres physiques utilisés pour le calcul numérique.

\begin{tabular}{lll}
\hline Paramètre & Unité & Valeur \\
\hline$\rho_{f}$ & $\mathrm{~kg} / \mathrm{m}^{3}$ & 1060 \\
$\mu_{f}$ & $c P$ & 320 \\
$\rho_{p}$ & $\mathrm{~kg} / \mathrm{m}^{3}$ & 2230 \\
$d p$ & $\mathrm{~mm}$ & 1.1 \\
$h_{m}$ & $\mathrm{~m}$ & $2.345 e-02$ \\
$h_{f}$ & $\mathrm{~m}$ & $6.247 \mathrm{e}-03$ \\
\hline
\end{tabular}

Afin de minimiser le temps de calcul, l'intégralité du domaine expérimental n'est pas reproduite. Le domaine de calcul a une longueur de $33 \mathrm{~mm}$ (i.e. 30dp), une largeur de $6.6 \mathrm{~mm}$ (i.e. $6 \mathrm{dp}$ ) et des conditions de périodicité sont appliquées en entrée et en sortie. Le lit sédimentaire d'épaisseur $h_{\text {bad }}$ est composé d'une couche granulaire mobile $h_{m}$ et fixe $h_{p}$. Afin de reproduire les conditions expérimentales, la paroi supérieure du domaine est fixée à la position $x_{e}=-h_{b e d}+h_{f}$, ce qui implique que la couche granulaire 


\section{Thème 2 - Dynamique sédimentaire}

fixe est inférieure à celle utilisée expérimentalement. Le plus important dans cette démarche est de s'assurer d'une part que $h_{\text {bad }}$ est bien supérieur à la couche mobile et, d'autre part, que l'épaisseur de la couche fixe restante est suffisamment importante pour ne pas affecter le déplacement de la couche mobile. Dans les résultats que nous présentons ici, la couche fixe est égale à environ $7.92 \mathrm{~mm}$ (i.e. 7.2dp), ce qui permet de satisfaire la condition citée précédemment.

La vitesse fluide calculée analytiquement par AUSSILLOUS et al. (2013) est utilisée pour mettre les sédiments en mouvement. Cette vitesse est décomposée en trois régions: une région pure fluide ne contenant aucune sphère où le profil vertical de vitesse est parabolique, une région mouvante où 1 a vitesse dépend de la concentration des sédiments et une région fixe où la vitesse est nulle.

Le domaine de calcul est discrétisé spatialement avec des cellules de taille $\Delta \mathrm{x}=6 \mathrm{dp}$, $\Delta \mathrm{y}=3 \mathrm{dp}$ et $\Delta \mathrm{z}=1.2 \mathrm{dp}$. On s'intéresse au profil vertical (moyenné sur les autres directions) de la fraction solide $\phi$ (voir figure 1) et de la vitesse des deux phases $\mathrm{U}$ (voir figure 2).

La fraction solide est définie par la relation :

$\phi=\frac{\sum_{i=1}^{n p} V_{p i}}{V_{\text {cell }}}$

où $n p$ représente le nombre de sphères de volume $V_{p i}$ contenues dans la cellule fluide $V_{\text {colll}}$. Le centre d'une sphère est utilisé pour détecter la cellule fluide contenant la sphère. Lorsqu'une sphère chevauche deux cellules fluides, le volume total de la sphère est associé à la cellule fluide contenant le centre de la sphère sauf dans la direction verticale où le volume exact de chaque découpe est associé à chacune des cellules fluides.

Les résultats présentés sont obtenus après 20 secondes de simulation et avec un pas de temps de $10^{-7} \mathrm{~s}$. On observe sur la figure $1 \mathrm{q}$ ue la simulation numérique arrive à reproduire assez bien les observations à l'exception du passage entre la phase fluide et la phase granulaire. Nous pensons que ce problème pourrait provenir des forces de lubrification entre les sphères, qui sont importantes ici, et qui ne sont pas prises en compte dans le modèle de contact. Le résultat de la figure 2 montre également que la simulation permet de retrouver le profil de vitesse des deux phases ainsi que les oscillations de vitesse présentes dans le lit dues à la présence des sédiments et observées expérimentalement. 


\section{XII ${ }^{\text {èmes }}$ Journées Nationales Génie Côtier - Génie Civil \\ Cherbourg, 12-14 juin 2012}

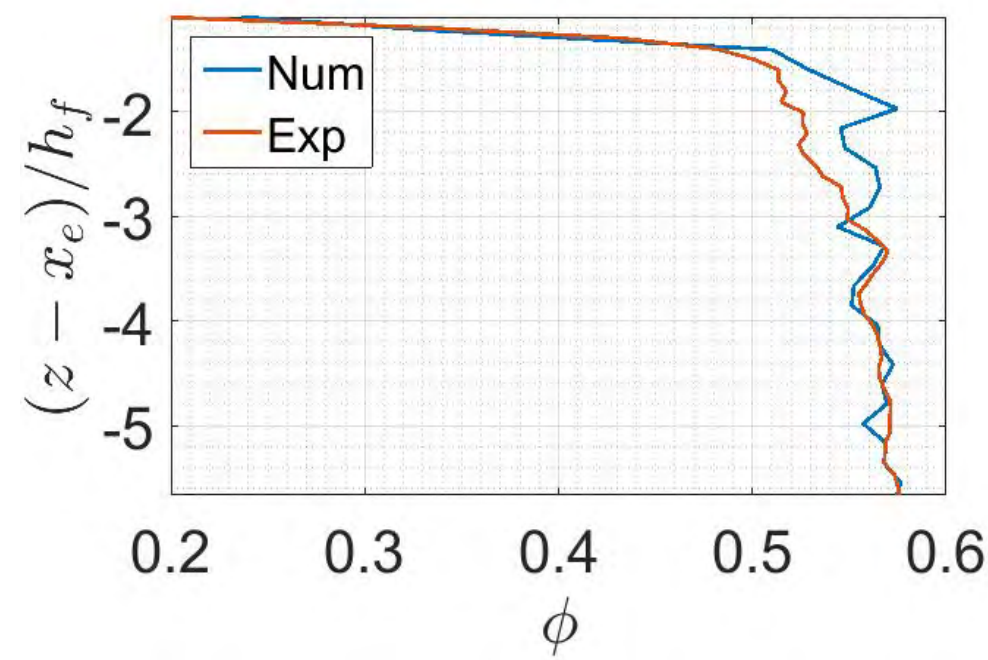

Figure 1. Comparaison entre les profils verticaux de la fraction solide obtenus expérimentalement (label Exp) et numériquement (label Num). L'axe vertical a été normalisé par la quantité $\left(\mathrm{z}-\mathrm{x}_{e}\right) / h_{f}$.

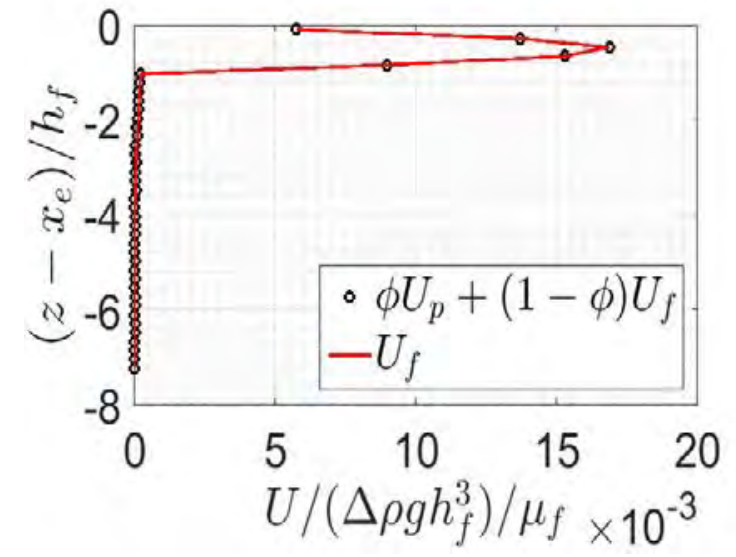

(a)

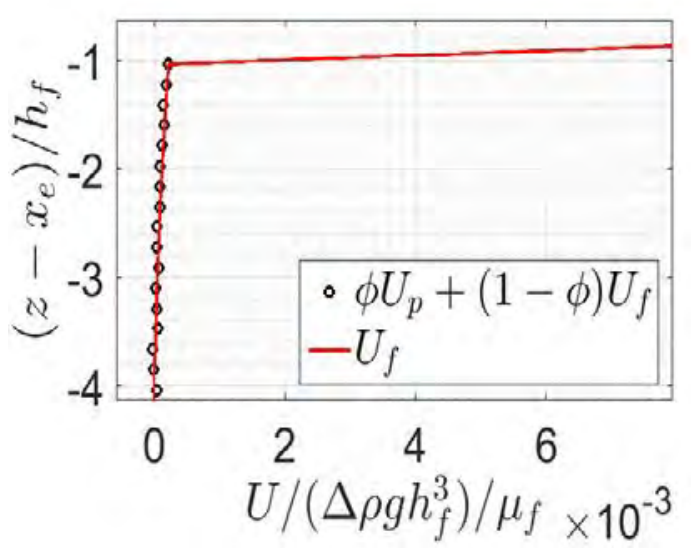

(b)

Figure 2. Comparaison entre le profil vertical de la vitesse fluide imposée (en rouge) et celui obtenu numériquement (cercle noir) : (a) variations sur l'intégralité de l'axe vertical, (b) zoom sur la zone de transition fluide-solide. L'axe vertical a été normalisé par la quantité $\left(z-\chi_{e}\right) / h_{f}$ et la vitesse par la quantité $\Delta \rho g h_{f}^{3} / \mu_{f}$ où $\Delta \rho$ est la différence des masses volumiques solide et fluide $\rho_{p}-\rho_{f}$ et g est l'accélération de gravité.

\section{Principe de forçage, cas test et application}

\subsection{Principe de forçage de YADE par MARS3D}

MARS est utilisé pour simuler les courants de marée dans le Nord Cotentin en vue des implantations futures des fermes pilotes hydroliennes. Il résout les équations primitives et utilise l'hypothèse hydrostatique, ainsi que l'approximation de Boussinesq. Ce modèle 


\section{Thème 2 - Dynamique sédimentaire}

est basé sur la méthode des différences finies et permet, via le module AGRIF, d'utiliser des grilles de calcul emboîtées afin d'affiner la solution numérique par l'intermédiaire d'une résolution plus fine sur certaines zones définies par l'utilisateur. Un schéma de type ADI est utilisé pour la discrétisation temporelle.

Le forçage de YADE par MARS a été réalisé à l'aide de l'interface Python de YADE. L'envoi des variables de MARS à YADE a lieu à chaque pas de temps de forçage. A l'instant initial, la bathymétrie de MARS $\left(h_{0}\right)$ est enregistrée dans YADE. Ensuite, MARS transmet à YADE l'élévation de la surface libre et la position des niveaux verticaux (respectivement $x_{e}$ et $\sigma_{w}$ ), qui sont utilisés pour détecter la cellule fluide associée au sédiment considéré ainsi que la vitesse des courants $3 \mathrm{D}, \overrightarrow{u_{f}}$, qui sert au calcul des forces de traînée et de portance.
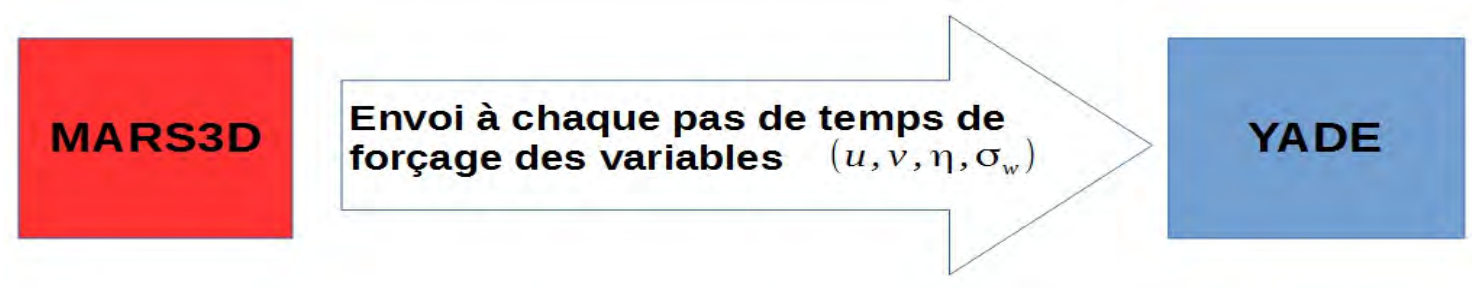

Figure 3. Schéma de forçage de YADE par MARS.

\subsection{Cas test de validation: Poiseuille sans gravité}

Afin de valider la procédure de forçage précédemment décrite, nous commençons par simuler un cas i déalisé qui a ét é réalisé dans les conditions suivantes : bathymétrie plane, gravité négligeable, et écoulement de Poiseuille en entrée et en sortie du domaine. L'objectif est de s'assurer que la trajectoire des sédiments en suspension est uniquement gouvernée par les caractéristiques du courant (direction et intensité).

Dans tout ce qui suit, les sédiments sont sphériques et de rayon $5 \mathrm{~cm}$ (ce qui correspond à des cailloutis). Ils sont composés de calcaire et se déplacent sur un fond en granite. Les résultats présentés sont obtenus avec un pas de temps de forçage de 2 minutes et un pas de temps dans YADE de l'ordre de $10^{-6} \mathrm{~s}$. Dans les différentes figures ci-après, les axes $\mathrm{x}$, y et z sont orientés respectivement du Sud vers le Nord, de l'Ouest vers l'Est et $\mathrm{du}$ fond vers la surface.

Le domaine de calcul a une longueur de $600 \mathrm{~m}$ en $\mathrm{x}$, une largeur de $300 \mathrm{~m}$ en y et une profondeur (hest) de $30 \mathrm{~m}$ en $\mathrm{z}$. La discrétisation spatiale est de $10 \mathrm{~m}$ en x et y. Vingt niveaux sigmas uniformes sont utilisés en z. Dans MARS un profil parabolique de type Poiseuille est imposé à l'instant initial, en entrée et en sortie du domaine. Le profil imposé est donné par :

$\overrightarrow{u_{f}}=\left(u_{x f}, 0,0\right) ; \quad u_{x f}=-4 \frac{U_{\max }}{\text { hest }^{2}} z(z+h c s t), \quad-h c s t \leq z \leq 0$. 


\section{XII ${ }^{\text {èmes }}$ Journées Nationales Génie Côtier - Génie Civil \\ Cherbourg, 12-14 juin 2012}

La valeur $U_{\max }=3 \mathrm{~m} / \mathrm{s}$ a été choisie pour s'approcher au mieux d'un environnement mégatidal extrême. Des conditions de périodicité sont utilisées sur les frontières latérales. De par la nature des conditions aux limites et initiales imposées, on obtient un profil de Poiseuille stationnaire donné par (3) et une élévation de la surface libre quasi nulle (de l'ordre de $10^{-4} \mathrm{~m}$ ) (cf. figure 4a). Nous disposons ensuite à l'entrée du domaine 1000 sédiments dans une boite de longueur $1 \mathrm{~m}$ en $\mathrm{x}$, de largeur $280 \mathrm{~m}$ en y et de hauteur $20 \mathrm{~m}$ en $\mathrm{z}$. La vitesse et la position des sédiments avant et après l'action de l'écoulement sont représentées sur les figures 4 (a)-(b)-(c). On constate que la trajectoire et la vitesse des sédiments est bien gouvernée par le champ de vitesse fluide, ce qui valide notre méthode de forçage.

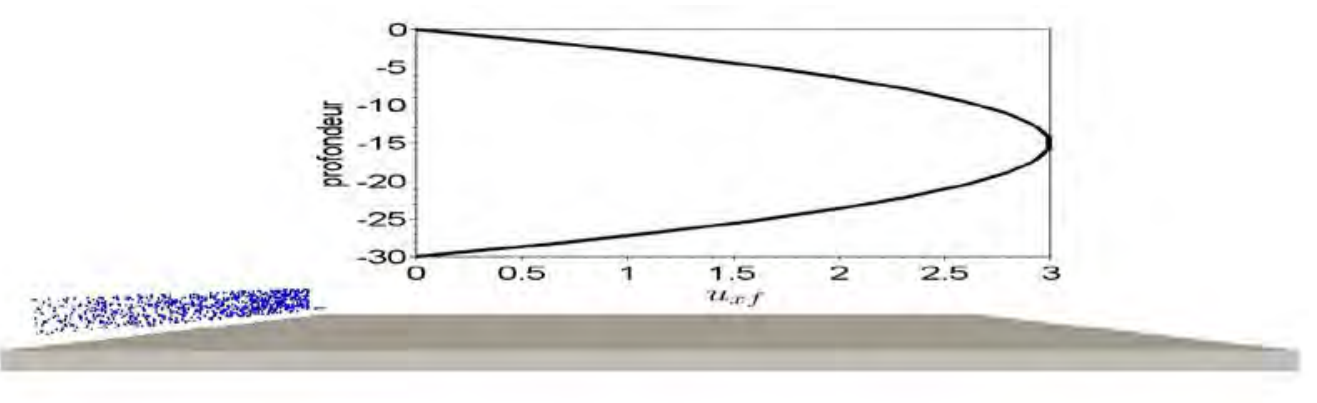

(a)

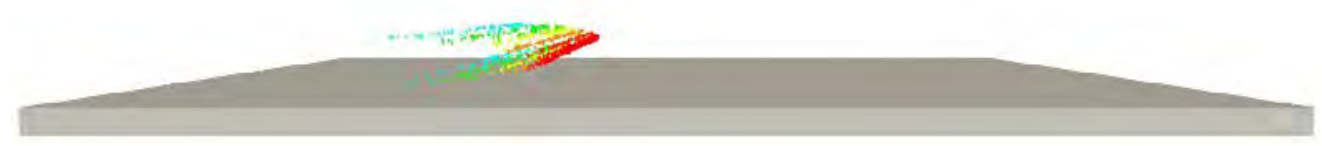

(b)

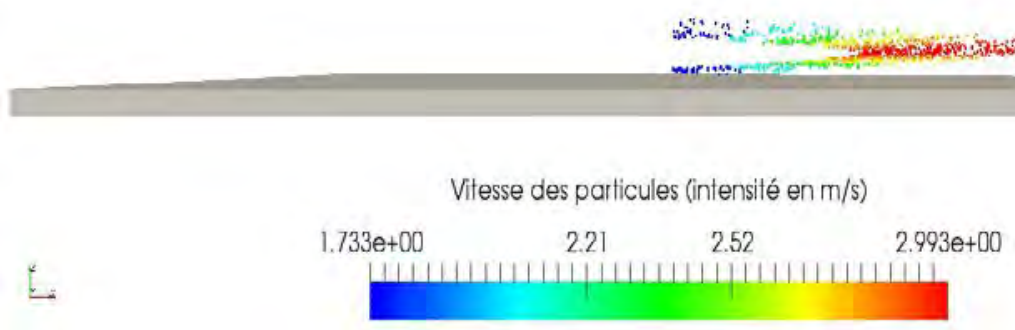

(c)

Figure 4. Position et vitesse des sédiments à l'instant initial (a), après 5 minutes de simulation (b) et 10 minutes de simulation (c).

\subsection{Application au Nord Cotentin}

La figure 5 montre la bathymétrie de la zone pour trois domaines emboités. Les simulations avec YADE sont effectuées sur le domaine le plus petit (à droite). La 


\section{Thème 2 - Dynamique sédimentaire}

résolution spatiale est de $10 \mathrm{~m}$ en longitude et latitude. Vingt niveaux sigmas non uniformes sont utilisés avec un $\mathrm{r}$ affinement près du fond. Les niveaux verticaux sont définis par l'équation (4).

$\sigma_{w}(k)=-1+\frac{1-Q^{k-1}}{1-Q^{k \max }}, \quad Q=r^{\frac{1}{k \max }}, \quad 1 \leq k \leq k \max +1$

où kmax est le nombre total de niveaux verticaux et $r$ le rapport entre l'épaisseur du dernier et du premier niveau fixé à $10, \mathrm{c}$ onduisant à une première couche verticale d'épaisseur variant de $0.49478 \mathrm{~m}$ à $0.78150 \mathrm{~m}$.

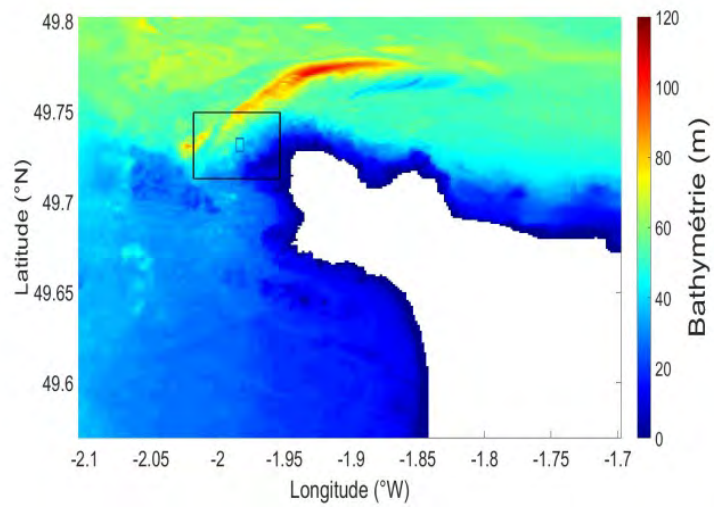

(a)

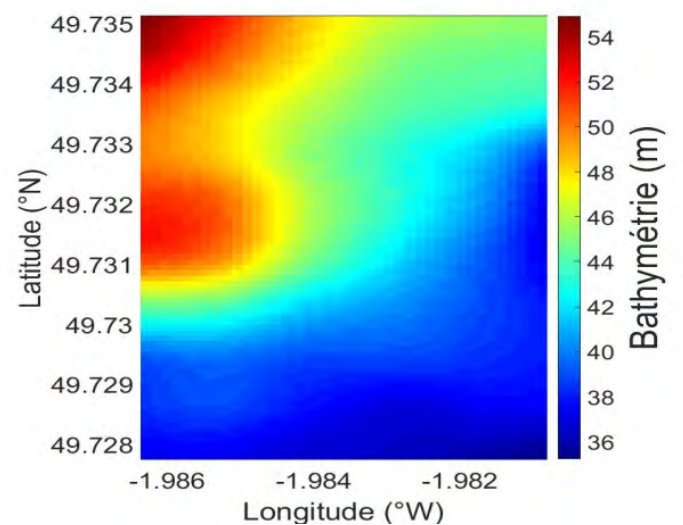

(b)

Figure 5. Variations bathymétriques des trois dernières grilles AGRIF utilisées (a) et de la grille la plus fine (b).

Nous disposons près du fond (i.e. dans la première cellule fluide) 1000 sédiments pour une superficie au sol de $20 \mathrm{~m}$ x $200 \mathrm{~m}$ (voir figure 7 (a)). La direction et l'intensité des courants près du fond ainsi que les profils verticaux de vitesse à l'instant initial sont représentés sur la figure 6. Compte tenu de la taille des sédiments (sphères de rayon $5 \mathrm{~cm}$ ) et de la vitesse du courant près du fond (compris entre $0.1787 \mathrm{~m} / \mathrm{s}$ et $0.8476 \mathrm{~m} / \mathrm{s}$ ), 14 minutes de simulation sont suffisantes pour observer le déplacement des sédiments sur l'ensemble du domaine.

Durant la simulation, on obs erve d'abord des zones d'accélération causées par la descente des pentes essentiellement localisées à l'Ouest du domaine (voir la figure 7 (b)). Par la suite, le déplacement des sédiments se scinde en deux zones : une première où les sédiments sont fortement accélérés par la pente située au Nord-Ouest (voir figure 5 ) et une seconde localisée sur la partie Nord-Est où l'effet des courants et des variations bathymétriques semble être plus équilibré (voir figure 7(c)). Pour éviter d'éventuels problèmes de bord, tout sédiment qui franchit les limites du domaine n'est pas réinjecté mais exclu de la simulation. Ainsi, la décomposition précédemment décrite décroît avec l'évolution du temps jusqu'à l'obtention d'un déplacement global plus homogène (voir figure 7(d)). 


\section{XII ${ }^{\text {èmes }}$ Journées Nationales Génie Côtier - Génie Civil \\ Cherbourg, 12-14 juin 2012}

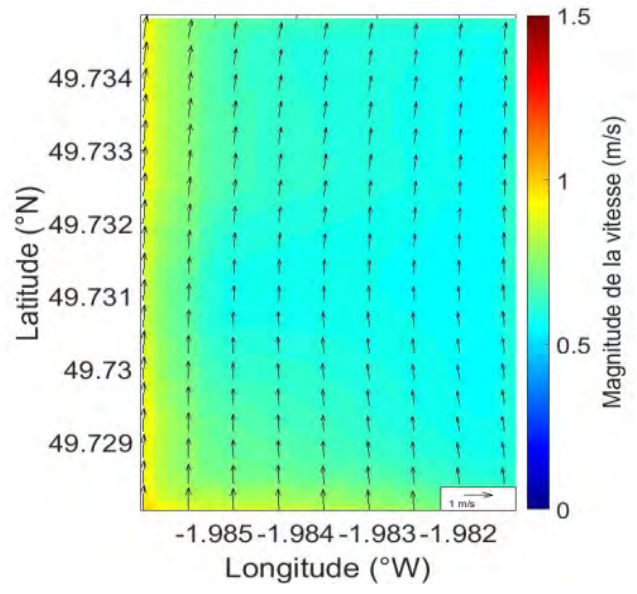

(a)
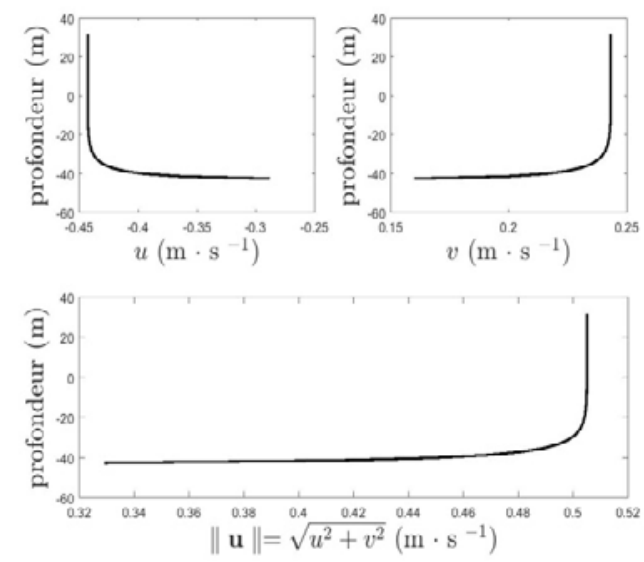

(b)

Figure 6. Direction et intensité des courants près du fond à l'instant initial (a).Profils verticaux des composantes du courant au centre du domaine. u représente la vitesse zonale et $v$ la vitesse méridionale.

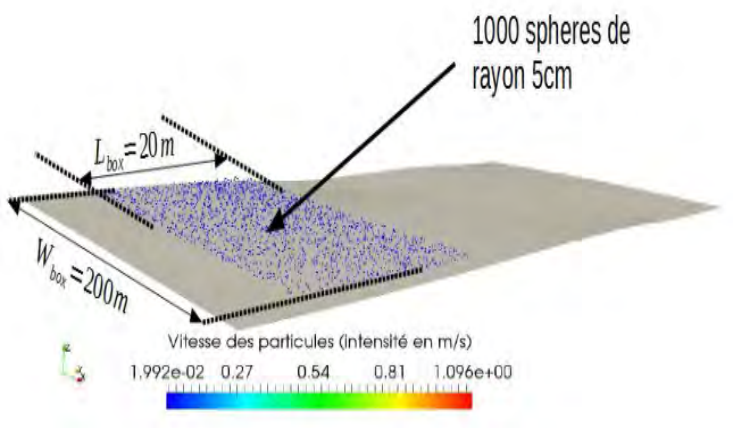

(a)

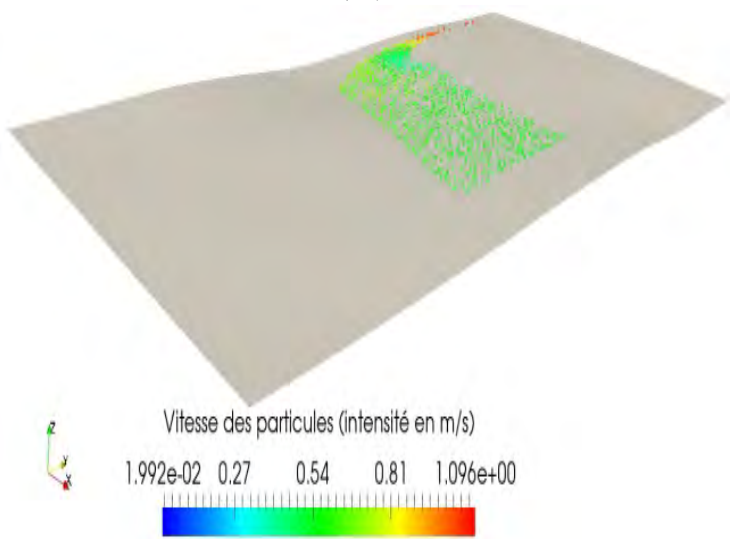

(c)

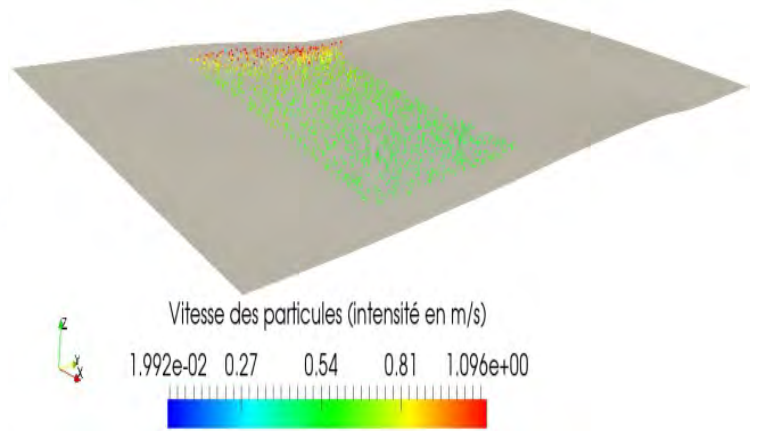

(b)

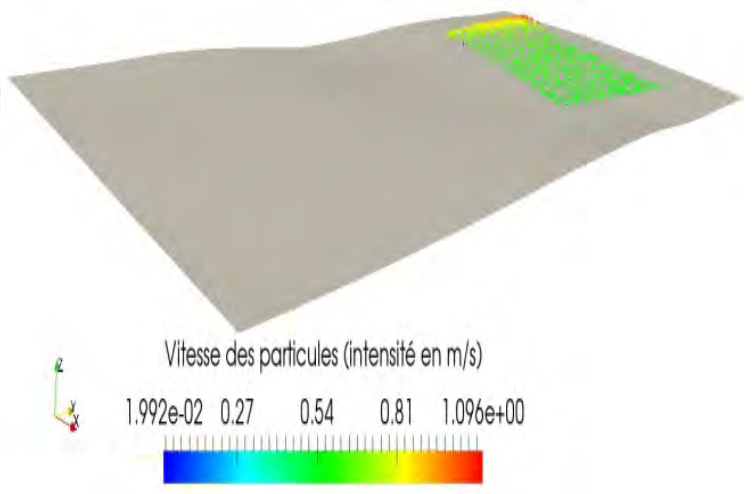

(d)

Figure 7. Vitesse et position des sédiments à l'instant initial (a), après 5 minutes (b), 8 minutes (c) et 14 minutes (d) de simulation. 


\section{Conclusions}

Les modifications apportées à Y ADE (généralisation au cas t ridimensionnel et implémentation d'une coordonnée verticale adimensionnée) pour un forçage par MARS sont validées à l'échelle du processus par comparaison avec les résultats expérimentaux de AUSSILLOUS et al. (2013). Les profils verticaux de la fraction solide et de la vitesse sont en assez bon accord avec les résultats expérimentaux. Les biais observés étant probablement dus à l'utilisation d'un fluide très visqueux pour les expériences, la validation peut être considérée comme effective pour l'application de YADE à l'échelle côtière où la lubrification n'intervient pas. La méthode de forçage de YADE par MARS a été présentée. Elle fait intervenir les forces de portance, de flottabilité et de traînée. La force de trainée a été modifiée par rapport au travail initié par MAURIN et al. (2015) pour prendre en compte des nombres de Reynolds de particule plus élevés. Les modifications étant validées, les premières simulations pour le Nord Cotentin ont été réalisées afin de les appliquer in-fine aux sites EMR hydrolien. On observe alors que le déplacement des sédiments près $d u$ fond est autant gouverné par l'intensité et la direction du courant que par les variations bathymétriques et les propriétés des sédiments. Afin d'affiner les résultats de YADE à 1 'échelle du processus, il serait intéressant d'introduire les forces de lubrification dans la loi de contact et quantifier son impact sur les résultats numériques. Pour l'application en milieu côtier, les perspectives les plus intéressantes seraient d'ajouter les effets de l'état de mer sur le courant de marée et d'utiliser un agrégat de sphères pour modéliser des sédiments de formes plus réalistes.

\section{Références bibliographiques}

AUSSILlOUS P., CHAUCHAT J., PAILHA M., MEDALE M., GUAZZELLI E. (2013). Investigation of the mobile granular layer in bedload transport by laminar shearing flow. J.Fluid Mech. Vol. 736, pp 594-615.http://dx.doi.org/10.1017/jfm.2013.546

DI FELICE R. (1994). The voidage function for fluid-particle interaction systems. Intl J. Multiph. Flow, Vol. 20, pp 153-159. http://dx.doi.org/10.1016/0301-9322(94)90011-6

LAZURE P., DUMAS F. (2008). An external-internal mode coupling for a 3D hydrodynamical model for applications at regional scale (MARS). Advances in Water Resources, Vol. 31(2), pp 233-250. http://dx.doi.org/10.1016/j.advwatres.2007.06.010

MAURIN R., CHAUCHAT J, CHAREYRE B., AND FREY P. (2015). A minimal coupled fluid-discrete element model for bedload transport. Phys. Fluids, Vol. 27, 113302. http://dx.doi.org/10.1063/1.4935703

ŠMILAUER V., A. GLADKY A., KOZICKI J., MODENESE C., STRANSKY J. (2010). Yade, using and programming. In Yade Documentation(Ed. V. Šmilauer), The YadeProject, 1st ed.

WIBERG P.L., SMITH J. D. (1985).A theoretical model for saltating grains in water. J. Geophys. Res. B, Vol. 90(C4), pp 7341-7354. http://dx.doi.org/10.1029/JC090iC04p07341 